\title{
Study of the function of metabotropic P2Y receptors in mast cells
}

\author{
Haruhisa Nishi ${ }^{1}$, François Niyonsaba ${ }^{2}$
}

${ }^{\text {I}}$ Pharmacology, The Jikei University School of Medicine, Japan, ${ }^{2}$ Atopy (Allergy) Research Center, Juntendo University Graduate School of Medicine, Japan

Study of the function of metabotropic P2Y receptors in mast cells

Mast cells express multiple ligand-gated P2X and metabotropic P2Y purinergic receptors. The P2X $\mathrm{X}_{7}$ receptor is known as the most important subtype of the purinergic receptors for mast cell activation, however the functions of the metabotropic P2Y receptors expressed on mast cells are unclear. It was previously shown that extracellular purines enhance IgE receptor stimulation-induced histamine release in human lung mast cells (1). However, the mechanism of the enhancement is unknown. The present study analyzed the mechanisms of P2Y receptors on mast cell activation using LAD2, a humanderived mast cell line. LAD2 were sensitized with/without anti-4-hydroxy-3-nitrophenylac acetyl antibody (anti-NP-IgE, $1 \mu \mathrm{g} / \mathrm{mL})$. Low level allergic stimulation was induced by NP-BSA $(1 \mathrm{ng} / \mathrm{mL})$. Purinergic agonists were added with/without allergic stimulation. None of the purinergic agonists in appropriate concentrations for P2Y receptor activation $(<1 \mathrm{mM})$ induced degranulation in LAD2 without allergic stimulation. Some purinergic agonists in extremely high concentration $(>1 \mathrm{mM})$ induced low level LAD2 deregulation without allergic stimulation. Combining ATP $\gamma \mathrm{S}$, socalled P2 ${ }_{11}$ agonist, with allergic stimulation enhanced the degranulation observed in LAD2. No purinergic agonist, including ATP $\gamma \mathrm{S}(0.1 \mathrm{mM})$, increased the weak allergic stimulation-induced intracellular $\mathrm{Ca}^{2+}$ mobilization which is one of the key components for degranulation in LAD2. Both phosphoinositide 3-kinase (PI3K) inhibitor and knockdowntreatment decreased the effect of purine agonist-induced enhancement of allergic stimulation-induced degranulation. Low level allergic stimulation with ATP $\gamma \mathrm{S}$ enhanced the phosphorylation of PI3K and protein kinase B (Akt), while the phoshoinositide-dependent kinase-1 (PDK1) did not. The effects of ATP $\gamma \mathrm{S}$ were inhibited by NF157, a P2Y ${ }_{11}$ antagonist in a dose-dependent manner. These results suggest that at least one subtype of P2YR is linked to enhancement of allergic degranulation in LAD2 not by $\mathrm{Ca}^{2+}$ mobilization but by PI3K-PIP2-Act pathway.

(1) Nishi,H., Pelleg A., Schulman, E.S., (2016) IgE Receptor-Mediated Histamine Release in Human Lung Mast Cells: Modulation by Purinergic Receptor Ligands Annals Clin Lab Sci 46: 463-469. 